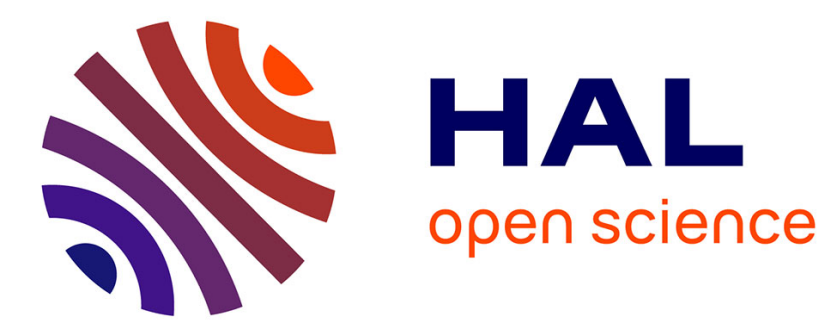

\title{
Adaptation of egocentric distance perception under telestereoscopic viewing within reaching space.
}

Anne-Emmanuelle Priot, Rafael Laboissière, Olivier Sillan, Corinne Roumes, Claude Prablanc

\section{- To cite this version:}

Anne-Emmanuelle Priot, Rafael Laboissière, Olivier Sillan, Corinne Roumes, Claude Prablanc. Adaptation of egocentric distance perception under telestereoscopic viewing within reaching space.. Experimental Brain Research, 2010, 202 (4), pp.825-36. 10.1007/s00221-010-2188-4 . hal-00686059

\section{HAL Id: hal-00686059 \\ https://hal.science/hal-00686059}

Submitted on 6 Apr 2012

HAL is a multi-disciplinary open access archive for the deposit and dissemination of scientific research documents, whether they are published or not. The documents may come from teaching and research institutions in France or abroad, or from public or private research centers.
L'archive ouverte pluridisciplinaire $\mathbf{H A L}$, est destinée au dépôt et à la diffusion de documents scientifiques de niveau recherche, publiés ou non, émanant des établissements d'enseignement et de recherche français ou étrangers, des laboratoires publics ou privés. 


\section{Adaptation of egocentric distance perception under 3 telestereoscopic viewing within reaching space}

\author{
4 Anne-Emmanuelle Priot • Rafael Laboissière • \\ 5 Olivier Sillan - Corinne Roumes • Claude Prablanc
}

6 Received: 30 July 2009/ Accepted: 4 February 2010

7 (C) Springer-Verlag 2010

8 Abstract Telestereoscopic viewing provides a method to 9 distort egocentric distance perception by artificially 10 increasing the interpupillary distance. Adaptation to such a 11 visual rearrangement is little understood. Two experiments 12 were performed in order to dissociate the effects of a 13 sustained increased vergence demand, from those of 14 an active calibration of the vergence/distance mapping. 15 Egocentric distances were assessed within reaching space 16 through open-loop pointing to small targets in the dark. 17 During the exposure condition of the first experiment, 18 subjects were instructed to point to the targets without 19 feedback, whereas in the second experiment, hand visual 20 feedback was available, resulting in a modified relationship between vergence-specified distance and reach distance. The visual component of adaptation in the second experiment was assessed on the unexposed hand. In the post-tests of both experiments, subjects exhibited a constant distance overestimation across all targets, with a more than twice larger aftereffect in the second one. These findings suggest

A.-E. Priot · C. Roumes

Institut de recherche biomédicale des armées (IRBA), Brétigny-sur-Orge, France

A.-E. Priot $\cdot$ R. Laboissière $\cdot$ C. Prablanc

INSERM, UMR-S 864, Espace et Action, Bron, France

A.-E. Priot $\cdot$ R. Laboissière $\cdot$ C. Prablanc

Université Claude Bernard Lyon 1, Lyon, France

A.-E. Priot · R. Laboissière · C. Prablanc $(\square)$

Institut Fédératif des Neurosciences de Lyon (IFNL),

Lyon, France

e-mail: claude.prablanc@inserm.fr

O. Sillan

Hospices Civils de Lyon-IFNL, Plateforme Mouvement et Handicap, Hôpital neurologique, Lyon, France two different processes: (1) an alteration in the vergence effort following sustained increased vergence; (2) a calibration of the vergence/distance mapping uncovering the visual component of adaptation.

Keywords Adaptation . Vergence - Binocular . Reaching space $\cdot$ Egocentric distance perception

\section{Introduction}

Reaching forward to grasp an object or to point to a target requires one to correctly evaluate its distance and direction. Egocentric distance is estimated from retinal and extraretinal cues (Gogel and Tietz 1979; Cutting and Vishton 1995; Cutting 1997; Genovesio and Ferraina 2004; Blohm et al. 2008). In order to get insight into the way the central nervous system (CNS) builds a body-centered representation of objects within near space, random sensory alterations can be introduced (Goodale et al. 1986; Prablanc and Martin 1992; Desmurget et al. 1999; Prablanc et al. 2003) as well as a continuous and systematic exposure to sensory alterations (Held and Freedman 1963; Prablanc et al. 1975; Kornheiser 1976; Kitazawa et al. 1997; Morton and Bastian 2004; Mon-Williams and Bingham 2007). In the latter case, the CNS can adapt to the new inter-sensory coupling in order to build up a coherent and unified representation.

The present study aimed at understanding how the estimation of egocentric distance is affected by exposure to systematic visual alteration, namely the wearing of a telestereoscope. A telestereoscope is a simple device composed of two pairs of lateral, parallel mirrors placed in front of the eyes of the subject, artificially increasing the interpupillary distance (IPD). As early as the seventeenth century, Kepler and Descartes proposed that the radial

\begin{tabular}{|l|lll|}
\hline Journal : Large 221 & Dispatch : 13-2-2010 & Pages : $\mathbf{1 2}$ \\
Article No. : $\mathbf{2 1 8 8}$ & $\square$ LE & $\square$ TYPESET \\
MS Code : EBR-09-0580 & $\sim_{\text {CP }}$ & $\checkmark$ DISK \\
\hline
\end{tabular}


distance to the point of fixation could be inferred from a triangulation process by using the convergence angle of the two lines of sight and the known IPD (Wade 1998). In this case, manipulation of the IPD would result in predicted modifications of egocentric distance estimation as vergence demand is modified. Artificially increasing the IPD by a given multiplicative factor of $\mathrm{N}$ increases the tangent of required convergence angle for all viewing distances by the same factor. Following this geometrical interpretation, Helmholtz proposed that "subjects viewed an exact reduced scale model of the world" through the telestereoscope. Increasing the IPD by a factor of $N$ would scale down the apparent distances by the same factor (Helmholtz 1910; Valyus 1966).

Fisher and Ciuffreda (1990) conducted the first experiment on adaptation of egocentric distance perception under telestereoscopic viewing. Subjects assessed the distance and depth of a pyramidal target located within reaching space $(33 \mathrm{~cm})$ before and after a 30-min period of telestereoscopic exposure involving locomotion and visuomotor activities. A perceptual aftereffect consisting of increased apparent target distance and depth was observed, concomitant with an increase in tonic vergence state.

The wearing of opposite-base prisms is an alternative way to modify the relationship between vergence and perceived distance. Prolonged exposure to prisms is known to induce adaptation with corresponding distance estimation aftereffects when viewing is restored to normal (Wallach and Frey 1972; Wallach et al. 1972; Wallach and Smith 1972; Craske and Crawshaw 1974; von Hofsten 1979; Owens and Leibowitz 1980; Ebenholtz 1981). Two main factors have been proposed to explain these aftereffects. The first one is a calibration of the mapping between vergence signal and perceived distance (referred hereafter as the calibration of the vergenceldistance mapping) arising from the conflict between altered vergence signal and unaltered monocular cues such as linear perspective, motion parallax or familiar size (Wallach and Frey 1972). The second one is a tonic change in the eyes muscles or eye muscle potentiation (EMP) (Ebenholtz 1974; Ebenholtz and Wolfson 1975; Paap and Ebenholtz 1977; Ebenholtz 1981; Ebenholtz and Fisher 1982). Both factors could be responsible for aftereffects, depending on exposure conditions (Welch 1986; Howard and Rogers 2002).

Vergence demands differ with regard to the optical device used. Prisms introduce a constant bias in the required convergence angles over all distances, whereas increasing the IPD by a given multiplicative factor with a telestereoscope increases the tangent of required convergence angle for all viewing distances by the same factor. In the present study, the adaptation of egocentric distance perception to telestereoscopic viewing within reaching space was investigated. Such an adaptive process may arise primarily from two components: an induction component induced by sustained fixation through the telestereoscope, and a calibration component based on distorted hand visual feedback. In order to disentangle these components, we designed two experiments differing only by the feedback given to the subject during exposure. In the pre- and posttests of both experiments, egocentric distance was estimated by open-loop pointing (i.e. without visual feedback of the hand) to the perceived location of the targets.

In Exp. 1, perceived distance under telestereoscopic viewing exposure was assessed by open-loop pointing. Throughout this paper, the distance given by the pointing gesture is referred as reach distance. Care was taken to limit the available cues for distance to the altered vergence during telestereoscopic viewing exposure. In that case, the expected aftereffect in Exp. 1 should originate mainly from the EMP mechanism. In order to ensure this, we designed the experiment such that the farthest target would appear at a distance of $195 \mathrm{~mm}$ from the eyes, which is below the point of balance between the actions of the medial and lateral recti muscles, called the physiological point of rest (PPR) (Ebenholtz and Wolfson 1975). The empirical value of the PPR is close to $300 \mathrm{~mm}$ (Paap and Ebenholtz 1977). Consistent with previous studies, we expected an EMPrelated increase in perceived distance over the whole range of targets after removal of the telestereoscope.

The goal of Exp. 2 was to study the adaptive processes arising from active visuomotor exposure to the telestereoscope. Held (1965) has shown that active experience is a key factor for perceptual adaptation to laterally displacing prisms. Active interaction with the environment also resulted in greater adaptation to prism-induced alteration of apparent distances (Owens and Leibowitz 1980; Ebenholtz 1981). In the exposure phase of Exp. 2, subjects underwent a discrepancy between vergence-specified distance and reach distance. Such a conflict may elicit visuomotor adaptation, whatever the specific contributions (motor, proprioceptive and visual) of the different adaptive components might be (Kornheiser 1976; Welch 1986; Redding and Wallace 1990). The present study focused on the visual component of adaptation only and investigated the potential calibration of the vergence/distance mapping as measured from the unexposed hand. Indeed, visuomotor adaptation is restricted to the exposed hand, whereas only the visual component of adaptation is available to the unexposed hand (Harris 1965). Comparable amounts of EMP are likely to be induced in both experiments, since these experiments differed only by the presence of a visual feedback. Calibration of the vergence/distance mapping requires the presence of this kind of feedback, whereas EMP does not. Any difference in the aftereffects of Exp.1 and 2 should thus be attributed to a calibration process. After removal of the telestereoscope, any theoretical

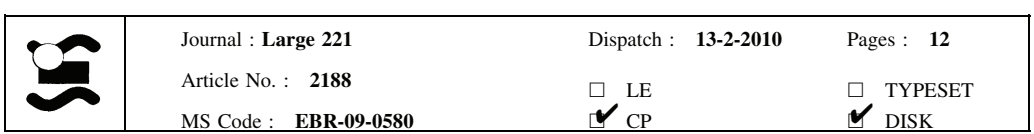




\section{Materials and methods} tion-related aftereffect.

\section{Subjects} between experiments. rescaling should result in an increase in the gain of the vergence/distance mapping, and thus a distance overestimation aftereffect. Hence, the aftereffect of Exp. 2 is expected to be larger than the aftereffect of Exp. 1, as it involves both the EMP-related aftereffect and the calibra-

All 24 recruited subjects gave informed consent. The experiments were conducted in accordance with the Declaration of Helsinki and under the terms of local legislation. All subjects were screened for good stereoscopic vision and none had past history of binocular disorder. All subjects had normal or corrected-to-normal vision. Prescribed correction, if any, was worn during the experiments. All subjects had to maintain single and clear vision over the whole range of targets by the end of the training phase. Twelve subjects were retained in Exp. 1 (six women and six men, mean age 38, ranging from 21 to 64) and twelve subjects were retained in Exp. 2 (six women and six men, mean age 34, ranging from 21 to 64). Five subjects participated in both experiments, with at least a 2-week delay

\section{Apparatus and procedure}

Figure 1a presents the telestereoscope and the optical path through telestereoscopic viewing. The telestereoscope consisted of two pairs of mirrors positioned parallel to each other, angled at $45^{\circ}$. The telestereoscope used in our experiments displaced the line of sight of each eye laterally by $70 \mathrm{~mm}$. The tangent of required convergence angle was thus increased by approximately $N=3.2$ times for a subject with a 64-mm IPD while fixating an object within near space. It can be noted that the telestereoscope also increases the path length of the light rays, shifting the virtual image (optical eye-to-target distance) by $70 \mathrm{~mm}$ further away. This decreases the accommodation level. The ratio of convergence to accommodation is therefore increased. The relationship between $y_{v}$ (vergence-specified distance through telestereoscope) and $\mathrm{y}_{\mathrm{a}}$ (optical eye-totarget distance, i.e. accommodation-specified distance) is given by: $\mathrm{y}_{\mathrm{a}} / \mathrm{y}_{\mathrm{v}}=$ IPD'/IPD $=\mathrm{N}$.

We used as visual stimuli nine red light-emitting diodes (LED, $635 \mathrm{~nm}$ wavelength) located vertically above the subject's head (see Fig. 1b). As the subject observed the targets through a central half-silvered mirror tilted $45^{\circ}$ with respect to the vertical fronto-parallel plane, the ramp of LEDs appeared horizontal. Direct vision through the mirror could be prevented by an occluding screen placed behind the mirror. The targets were aligned 350 to $510 \mathrm{~mm}$ from the cornea along a horizontal axis in the sagittal plane, $20 \mathrm{~mm}$ below the ocular plane. Head movements were restrained using a forehead and a chin rest.

In all experiments, distance estimates were assessed by open-loop pointing (i.e. without visual feedback of the hand) with the right hand. Indeed, visual egocentric distance estimated by pointing response has been found to be half as variable than verbal estimation (Foley 1977; Bingham and Pagano 1998) and more accurate. While the assessment of target distances by verbal responses involves mainly the occipito-temporal connection (i.e. the ventral pathway), a direct hand pointing response, under full spatial compatibility between the stimulus and the effector, and free of physical constraints, involves essentially the dorsal occipito-parietal connection (i.e. the dorsal pathway), as proposed by Goodale and Milner (1992). Hand pointing distance estimation is rather robust and weakly sensitive to cognitive judgments. A 2-mm infrared-emitting diode (900 nm wavelength) was attached to the fingertip, whose position was recorded at $250 \mathrm{~Hz}$ with an Optotrak 3020, Northern Digital Inc., a system for recording 3D movement. During the experiments, all pointing movements were performed in a totally free open space preventing any tactile feedback.

In preliminary tests, distance estimation of familiar objects under telestereoscopic viewing in a natural environment was assessed through verbal judgment. We noticed that objects with familiar size led to some ambiguity in judging egocentric distances. Some subjects perceived the objects to be near, likely relying on increased convergence. Other subjects perceived them further, likely relying on the decreased apparent size of the object. In order to reduce such an effect, we used small (3-mm diameter) LED targets. Virtual targets were used to prevent tactile feedback. The apparatus was calibrated using the Optotrak. A LED marker was mechanically displaced until two experimenters on both right and left sides of the halfsilvered mirror judged it coincident with the target image seen through the mirror. We estimate the accuracy of this procedure to be smaller than $1 \mathrm{~mm}$.

\section{Experiment 1}

A classical paradigm including three blocked conditions, pre-test, exposure to telestereoscopic viewing and post-test (Helmholtz 1910; Held and Freedman 1963), was carried out in an otherwise dark room. In all pre-tests, post-tests and exposure phase, the estimated distance was assessed by open-loop pointing toward the targets seen through the tilted central mirror. Cues for target distance during the exposure condition were restricted to altered oculomotor

\begin{tabular}{|l|lll|}
\hline Journal : Large 221 & Dispatch : 13-2-2010 & Pages : $\mathbf{1 2}$ \\
Article No. : $\mathbf{2 1 8 8}$ & $\square$ LE & $\square$ TYPESET \\
MS Code : EBR-09-0580 & $\sim_{\text {CP }}$ & $\checkmark$ DISK \\
\hline
\end{tabular}




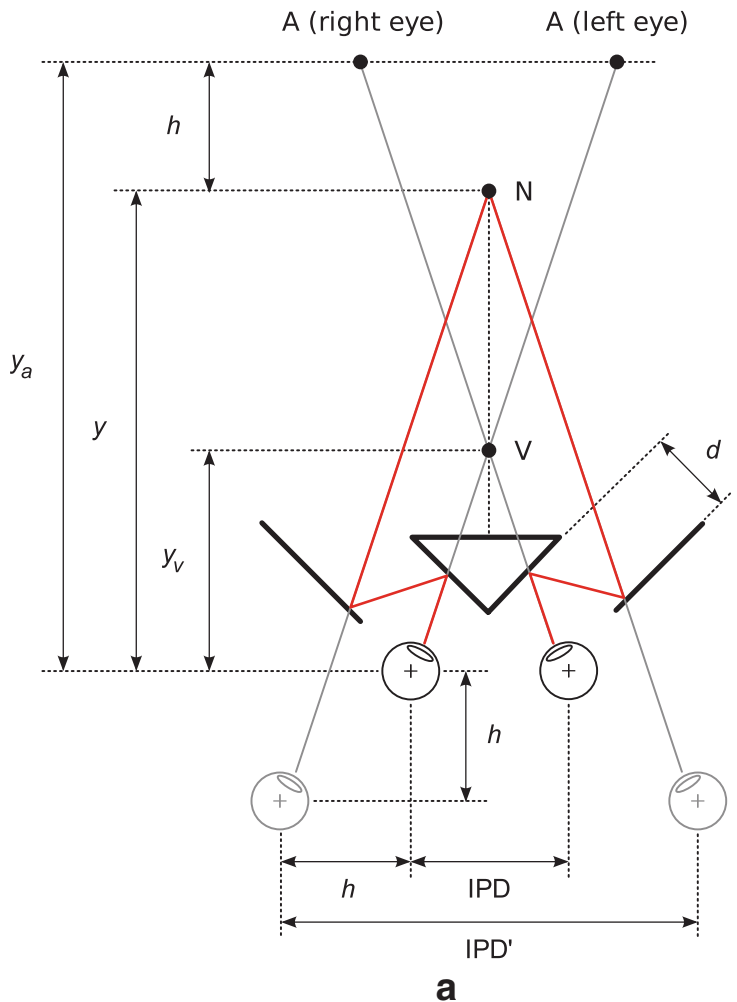

Fig. 1 a Telestereoscope geometry. Inner and outer vertical mirrors are parallel and slanted at $45^{\circ}$ from the sagittal plane. They are separated by an orthogonal distance $d$. The interpupillary distance is IPD and the apparent interpupillary distance IPD'. Virtual eyes are shown in gray and are displaced backwards and outwards by a distance $h=d \sqrt{ } 2$. The fixation point is represented by the dot labeled $\mathrm{N}$ (for "natural" viewing condition), placed at distance $y$ from the subject's eyes. Gray lines represent the direct lines of sight from the real and virtual eyes. The red line is the reflected light-path from the target to the eyes. Fixation at the dot labeled $\mathrm{N}$ requires the convergence of the two lines of sight onto the dot labeled $\mathrm{V}$, at cues (i.e. vergence and accommodation). This is an induction rather than an adaptation paradigm as no feedback was available during exposure (Ebenholtz 1981). We chose to use the same (right) hand for exposure and test conditions in Exp. 1 in order to compare pointing responses with and without the telestereoscope (i.e. to reveal the distortion of perceived distance).

Each of the nine targets was presented ten times in a random order. A single target only was lit at a time. There was a 3-s dark interval between offset of the previous target and onset of the next one, in order to reduce any existing inter-target disparity between two successive stimuli. Each condition lasted about $10 \mathrm{~min}$. Subjects underwent training trials without telestereoscope and without any feedback for a few minutes just before the pre-test, in order to become familiar with the pointing task. Before the exposure condition, subjects were trained under telestereoscopic viewing until they were able to fuse the targets at all distances.

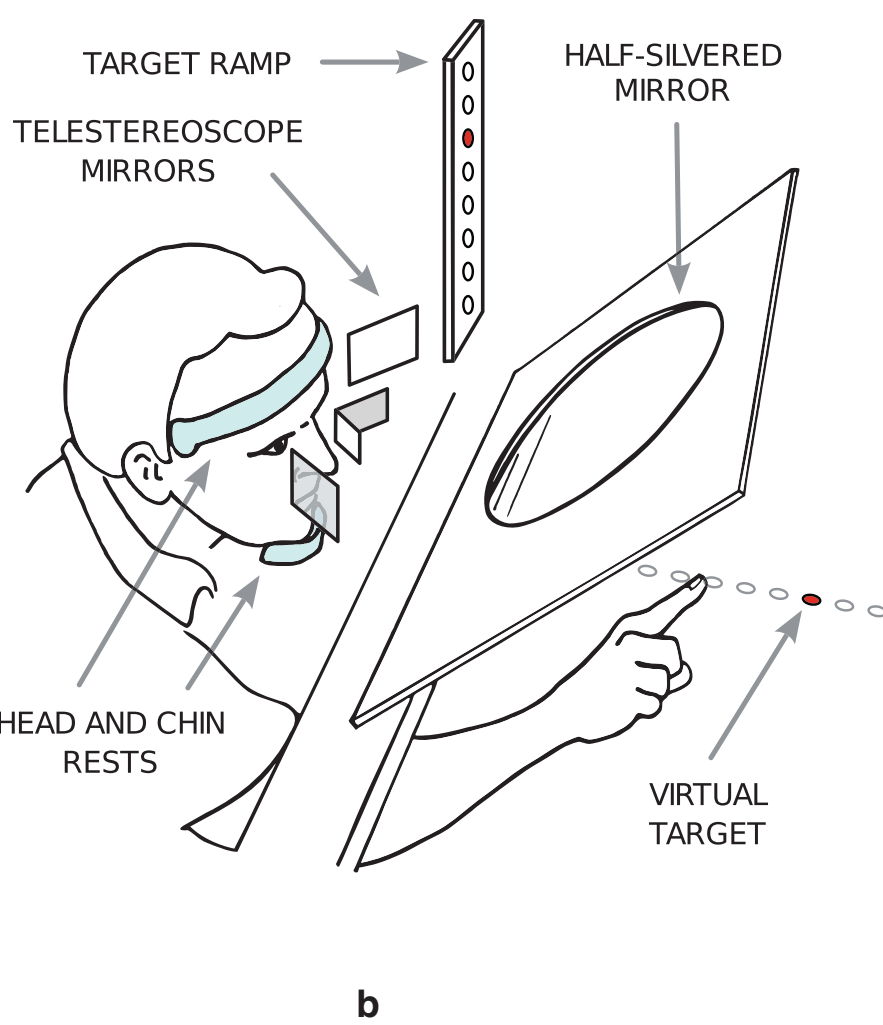

distance $y_{v}$ from the subject's eyes. The optical eye-to-target distance is $y_{a}$. The dots labeled A (right eye) and A (left eye) represent, respectively the virtual target images seen by right and left eyes. $y, y_{v}$ and $y_{a}$ correspond, respectively to the physical distance to the target, the distance as specified by vergence and the distance as specified by accommodation. b Experimental setup. The subject is looking through the telestereoscope mirrors while resting his head and chin. The pronated right hand points to the estimated position of the target behind the half-silvered mirror. The virtual position of the target is shown in red, as well as the lit LED in the target ramp

No pointing response was required during this training phase. Although most of the included subjects reported diplopia and/or blurring through the telestereoscope at the beginning of the training phase, they all reported single and clear vision over the whole range of targets by the end of the training phase.

In all conditions, subjects were instructed to point as accurately as possible with their unseen right index fingertip (moving in a free open space), at the perceived location of the target. Then, they validated the pointing response by pushing a button with the hanging stationary left hand, and returned the right hand to a rest position close to the chest. In the exposure condition, subjects were instructed to wait until they totally fused onto the target, before initiating their pointing response. In the rare instances when fusion was not possible (mostly for the nearest targets), the trial was aborted and the next random trial was presented. The aborted trials were presented after

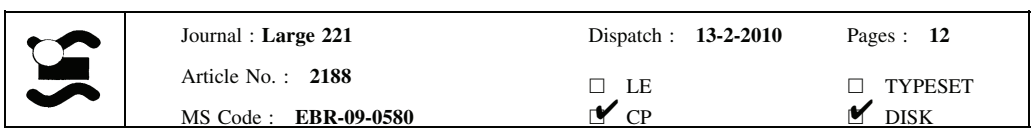


the end of the full trial sequence, intermixed with new random trials until ten repetitions of each target were collected. The post-test condition followed the exposure condition by a few minutes.

\section{Experiment 2}

The experimental setup was the same as in Exp. 1. The preand post-tests were carried out exactly in the same way as in Exp. 1, by open-loop pointing to the target with the right hand. In contrast to Exp. 1, the left hand was used for pointing during telestereoscopic viewing exposure, and hand visual feedback was allowed by removing the occluding screen behind the half-silvered mirror. Instead of directly seeing their left hand, subjects saw a red LED attached to the left index fingertip in an otherwise dark room in order to prevent hand or finger familiar size cue.

As in Exp. 1, subjects had to totally fuse onto the target before initiating the pointing response. They were instructed to point their left index finger as accurately as possible at the perceived location of the virtual target. Adjustment movements were allowed until the fingertip LED coincided with the virtual target. Then, the subject validated his or her response by pushing a button with the hanging and stationary right hand. Failure in binocular fusion resulted in trial abortion.

The left hand was used for pointing in the exposure condition of Exp. 2, whereas the right unexposed hand was used in the pre- and post-test conditions in order to isolate the visual component of aftereffect adaptation. In any type of visuomotor adaptation, the total aftereffect is a combination of visual, proprioceptive and hand motor components (Welch 1986). The two latter ones are restricted to the exposed limb. Indeed, previous studies on short-term visuomotor pointing adaptation to prism lateral displacement showed that these components are not transferred from the exposed limb to any other limb (Harris 1963, 1965; Hamilton 1964; Prablanc et al. 1975; Elliott and Roy 1981; Martin et al. 1996; Kitazawa et al. 1997). Conversely, when a visual component has developed, it is available for all effectors and an interlimb transfer is observed (Kornheiser 1976; Wallace and Redding 1979). Thus, using the unexposed right hand for distance assessment underscored the visual component of adaptation only, uncontaminated by the short-term visuomotor adaptation of the exposed left hand. Consequently, care was taken to avoid any contact between right and left hands throughout the experiment, which could have introduced some interlimb proprioceptive and/or motor transfer. It was necessary to run Exp. 2 after Exp. 1 for the five subjects enrolled in both experiments, in order to prevent any knowledge of result that would have been obtained during the Exp. 2 exposure.
Data analysis

The fingertip position in $3 \mathrm{D}$ was measured as the average over the $40 \mathrm{~ms}$ following the onset of the push button. For the data analysis, the measured variable was the pointing distance along a horizontal axis in the sagittal plane located $20 \mathrm{~mm}$ below the ocular plane. The origin of this axis lays at the coronal plane passing at the subject's cornea, making it appropriate for the assessment of egocentric distance estimation.

Inside a condition, the measured pointing distance depended on the target distance. To check for possible temporal drifts in the pointing behavior over the course of the open-loop conditions, we used a linear model that is described in the Appendix. Linear models are classically used to describe the functional relationship between target distance and distance estimation assessed by manual setting (Ebenholtz 1981; Mon-Williams and Tresilian 1999). The relationship between target distance and pointing distance is referred hereafter as the target-to-pointing mapping.

For each subject, an ANCOVA was performed to assess the aftereffect, using the pointing distance as dependent variable, the condition as a 2-level factor (pre- vs. post-test in both Exp. 1 and Exp. 2, and pre-test vs. exposure in Exp. 1), and the target distance as a continuous factor. The aftereffect is defined as the signed difference between post- and pre-test mean pointing distances (see Appendix). Repeated-measures ANCOVAs were performed on each group of subjects for Exp. 1 and Exp. 2. T-tests were performed to compare aftereffects in Exp. 1 and Exp. 2. To better understand the changes between the pre-test and the exposure conditions in Exp. 1, a MANOVA was performed using the mean pointing distance and the regression slope as dependent variables and pre-test vs. exposure condition as a 2-level factor.

\section{Results}

Preliminary analysis

For each experiment, we checked for the presence of outliers in the group of subjects, based on the global aftereffect. For each subject and for each experiment, the $z$-score was computed. Subjects whose $z$-score laid outside the \pm 2.0 interval were considered outliers and removed from the analysis. This happened only in Exp. 1 for one subject.

Check for possible temporal drifts in the pointing behavior over the course of the open-loop conditions was performed before averaging the data (see Appendix). Some subjects exhibited an increase in the mean pointing

\begin{tabular}{|c|c|c|c|c|}
\hline & Journal : Large 221 & Dispatch & 13-2-2010 & Pages: 12 \\
\hline & $\begin{array}{l}\text { Article No. : } \mathbf{2 1 8 8} \\
\text { MS Code : } \quad \text { EBR-09-0580 }\end{array}$ & $\begin{array}{l}\square \mathrm{LE} \\
\boldsymbol{V}_{\mathrm{CP}}\end{array}$ & & $\begin{array}{l}\square \text { TYPESET } \\
\boldsymbol{~ D I S K ~} \\
\end{array}$ \\
\hline
\end{tabular}


distance and/or the slope of the target-to-pointing mapping over the course of a condition block, while others exhibited a decrease. We ran two-sided $t$-tests on the set of values of time-coefficients for each experiment and for each condition, which showed that the group means were not significantly different from zero. The $t$-tests for the mean pointing distance time-coefficient had a minimum $P>0.14$ and the $t$-tests for the slope time-coefficient had a minimum $P>0.27$. We concluded that no systematic temporal trend was found in the group. For the remaining analysis in this paper, we will consider the subjects' responses to be stationary inside each condition block.

\section{Individual analyses}

In Fig. 2, pointing distance is plotted as a function of target distance for two different subjects, one in Exp. 1 (left panel) and the other in Exp. 2 (right panel). ANCOVAs between pre- and post-tests showed that the condition factor was significant for all subjects $(P<0.05)$, except for two subjects in Exp. 1 who presented aftereffects close to zero. The linear dependency on the target distance was, as one would expect, reliable for all subjects $(P<0.001)$ in both experiments. In Exp. 1, the fitted slope ranged from 0.45 to 1.15 , with mean value $0.81(\mathrm{SD}=0.20)$ in the pretest condition, and from 0.30 to 1.19 , with mean value 0.77 $(\mathrm{SD}=0.27)$ in the post-test condition. Values for Exp. 2 were similar, with the slope varying from 0.56 to 1.10 ,

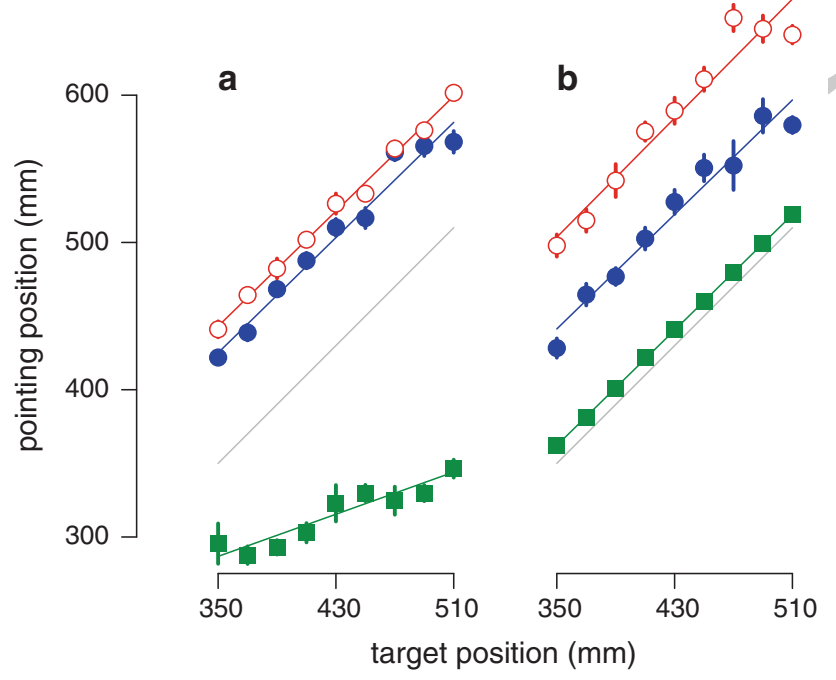

Fig. 2 Individual examples of distance estimation for Exp. 1 (panel a) and Exp. 2 (panel b) for two different subjects. In both panels, each point represents the average value of the pointing distance (vertical axis) for each target (horizontal axis), in each condition (blue-filled circles: pre-test, green-filled squares: exposure, red open circles: post-test). Standard errors are indicated by vertical bars. Regression lines for each condition are shown in the respective colors. The gray line indicates the ideal response under normal viewing mean value $0.85(\mathrm{SD}=0.18)$ in the pre-test, and from 0.55 to 1.19 , mean value 0.82 ( $\mathrm{SD}=0.20)$ in the post-test.

In Exp. 1, the interaction between condition and target distance was significant for three subjects, while in Exp. 2 it was significant for four subjects (maximum $P<0.05$ ). The individual aftereffect values for Exp. 1 and Exp. 2 are presented in Fig. 3. Each subject is represented by a point and the horizontal lines indicate the mean value for each experiment. The colored horizontal strips represent the standard errors on the estimation of the mean. In Exp. 1, the mean value of the aftereffect was $28 \mathrm{~mm}(\mathrm{SD}=34 \mathrm{~mm})$. The values ranged from $-18 \mathrm{~mm}$ to $104 \mathrm{~mm}$. In Exp. 2, the aftereffect averaged to $65 \mathrm{~mm}(\mathrm{SD}=36 \mathrm{~mm})$, with minimum and maximum values equal to $11 \mathrm{~mm}$ and $130 \mathrm{~mm}$, respectively. The aftereffect values computed for each subject will be used below in the $t$-tests.

\section{Group analysis}

The global behavior of the group of subjects in both experiments is shown in Fig. 4. This figure is similar to Fig. 2, but the points represent now the mean results for the group. Repeated-measures ANCOVA between pre- and post-test conditions showed significant results for the target distance factor $(F[1,10]=129, P<0.001$, in Exp. 1 , and $F[1,11]=247, P<0.001$, in Exp. 2). The condition factor was significant in both experiments $(F[1,10]=7.21$, $P<0.03$, in Exp. 1 , and $F[1,11]=40.4, P<0.001$, in

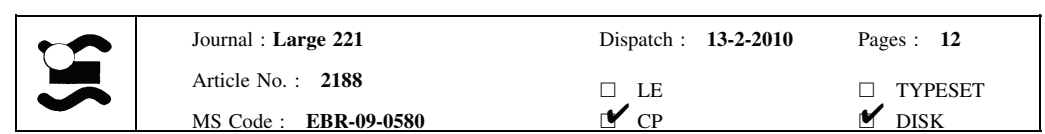

Fig. 3 Aftereffects in Exp. 1 and 2. The vertical axis represents the aftereffect, computed as the difference in the mean pointing distance from the pre- to post-test conditions. Each subject in each experiment is represented by a point (filled circles for Exp. 1 and open squares for Exp. 2). Horizontal lines show the mean value of the aftereffect in each experiment (solid line for Exp. 1 and dotted line for Exp. 2). Gray horizontal strips represent the \pm 1 SE interval of the estimation of the means

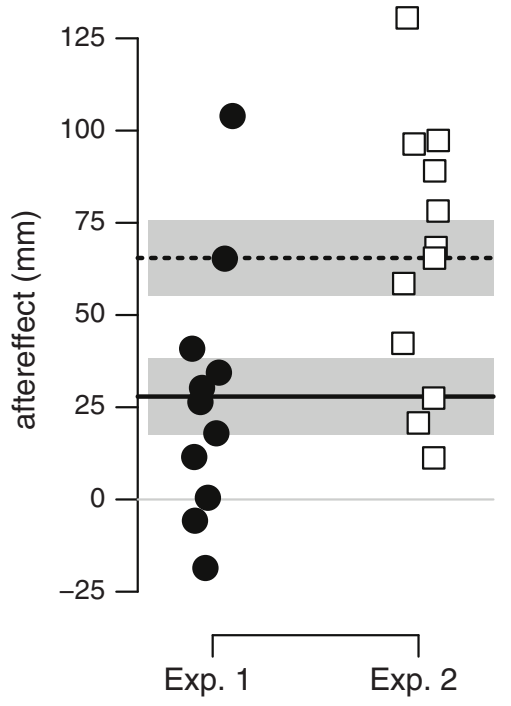




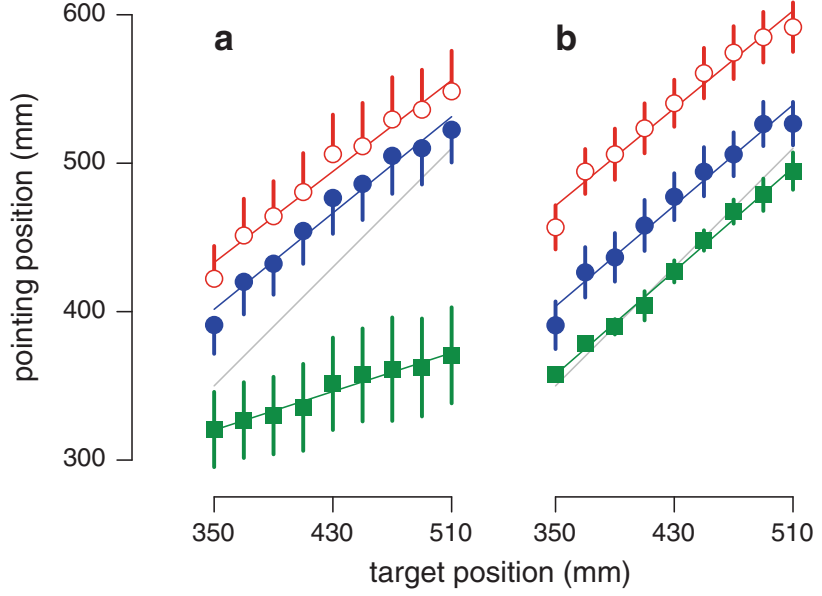

Fig. 4 Distance estimation mean across subjects, in Exp. 1 (panel a) and Exp. 2 (panel b). Conventions are the same as in Fig. 2 (bluefilled circles: pre-test, green-filled squares: exposure, red open circles: post-test). Standard errors are indicated by vertical bars. In the left panel, the error bars are plotted on one side only, for sake of clarity. Regression lines for each condition are shown with the corresponding color. The gray line indicates the ideal response under normal viewing
Exp. 2). The interaction between condition and target distance was not significant $(F[1,10]=0.72, P>0.41$, in Exp. 1, and $F[1,11]=1.19, P>0.3$, in Exp. 2). As it was shown in the previous section, the assumption of parallelism in the target-to-pointing regression lines between preand post-test failed for seven subjects out of 23. However, the lack of interaction between the condition and target distance factors as revealed by the group ANOVAs indicated that it is reasonable to assume such a parallelism at the group level.

One-sided paired $t$-tests were run on the aftereffect sizes for each experiment. The mean value of aftereffect in Exp. 1 was $28 \mathrm{~mm}(\mathrm{SE}=10 \mathrm{~mm})$ and significantly greater than zero $(t[10]=2.69, P<0.02)$. This was also the case for the mean value $65 \mathrm{~mm} \quad(\mathrm{SE}=10 \mathrm{~mm})$ in Exp. 2 $(t[11]=6.38, P<0.001)$. The difference in the aftereffect size across both experiments was assessed through an unpaired one-sided $t$-test. Exp. 2 has a significantly higher aftereffect than Exp. $1(t[21]=2.57, P<0.01)$.

\section{Exposure condition in Exp. 1}

To better understand the changes between the pre-test and the exposure conditions in Exp. 1, we conducted a multivariate analysis (MANOVA) on the mean pointing distance and slope. The data for the 11 subjects in Exp. 1 are shown in Fig. 5b. The theoretical curves for the natural viewing condition (N), as well as the vergence-specified (V) and accommodation-specified distance (A) are illustrated in Fig. 5a. The linear-regression coefficients of these theoretical curves are represented in Fig. 5b. They were computed assuming a mean IPD of $64 \mathrm{~mm}$ and a frontal separation between mirrors of $70 \mathrm{~mm}$ (see Material and methods). The MANOVA revealed a significant difference across conditions $(F[2,19]=10.7, P<0.001)$, indicating a difference in both mean pointing distance and slope between Exp. 1 and Exp. 2.

In the exposure condition of Exp. 1, the mean slope across subjects was 0.32 , with standard error equal to 0.07 . One-sided $t$-tests indicated that this value was significantly greater than zero $(t[10]=4.57, P<0.001)$ and lower that $1(t[10]=9.75, P<0.001)$. We compared this mean slope with the slope predicted by using the vergence information, which is 0.31 , through a two-sided $t$-test and no significant difference was found $(t[10]=0.13, P>0.89)$. The use of the vergence information also predicts that the mean pointing distance should be $157 \mathrm{~mm}$. The mean pointing distance for the group during the exposure condition of Exp. 1 was $314 \mathrm{~mm}$, with a $31-\mathrm{mm}$ standard error. This value was significantly greater than the value predicted $(t[10]=5.05, P<0.001)$. For all subjects, the mean pointing distance was greater than $157 \mathrm{~mm}$, with a 236-mm minimal value.

\section{Discussion}

Temporal evolution of target-to-pointing mapping

As in all adaptation or induction processes, there is both a rising acquisition function during the exposure phase and a corresponding post-exposure decline. This decline may be the result of a return to normal visual or visuomotor experience (i.e. de-adaptation), or the result of some spontaneous decay (Welch 1986). The spontaneous decay is much longer than the de-adaptation decay (Hamilton and Bossom 1964). The EMP-related aftereffect decay was expected to occur in both Exp. 1 and Exp. 2 post-tests, whereas the lack of visuomotor feedback in Exp. 2 post-test did not allow de-adaptation of the calibration of the vergence/distance mapping. In both experiments, these time constants should exist and be large enough in comparison with the duration of the condition blocks, otherwise we should have measured no aftereffect. However, the temporal analysis did not show a significant variation over time for the group of subjects, but a large inter-subject variability was found both in the amount and in the direction of the drift. Brown et al. (2003) and Wann and Ibrahim (1992) have already shown substantial amounts of proprioceptive drifts for reach movements. The expected adaptation- or induction-related temporal evolution may have been masked by the noise in open-loop hand pointing. Significant and significantly different aftereffects were obtained

\begin{tabular}{|l|lll|}
\hline & Journal : Large 221 & Dispatch : 13-2-2010 & Pages : $\mathbf{1 2}$ \\
Article No. : $\mathbf{2 1 8 8}$ & $\square$ LE & $\square$ TYPESET \\
MS Code : EBR-09-0580 & $\sim_{\mathrm{CP}}$ & $\checkmark$ DISK \\
\hline
\end{tabular}




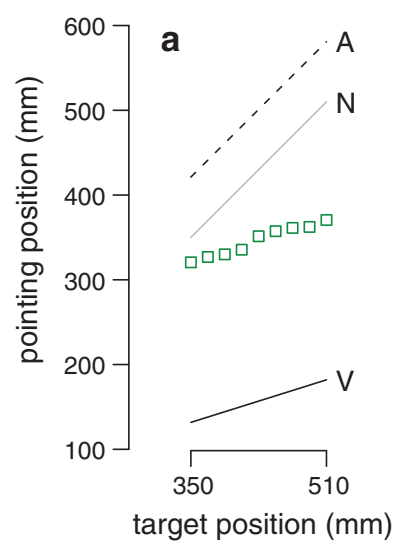

Fig. 5 a Theoretical responses using the different signals used for distance estimation under telestereoscopic viewing. The pointing distance (vertical axis) is plotted against the target distance (horizontal axis). The solid line represents the distance as specified by vergence $(V)$ and the dashed line shows the distance as specified by accommodation $(A)$ for a telestereoscope with a 70-mm frontal separation between mirrors. The gray line represents the physical distance to the target under natural viewing $(N)$. The mean group response for each target is shown with green open squares. b Change in the linear-regression coefficients between pre-test and exposure

in Exp. 1 and 2 despite all drifts and noise in open-loop pointing.

\section{Exp. 1: the induction paradigm}

The first aim of the present study was to investigate egocentric distance estimation under telestereoscopic viewing in a reduced visual cue environment without any feedback, and distance estimate alteration following sustained increased convergence.

The distance estimate aftereffect following the induction paradigm may be accounted for by changes in oculomotor adjustments, namely the increase in tonic vergence and/or increase in accommodative vergence gain. Vergencespecified distances ranged from $134 \mathrm{~mm}$ to $195 / \mathrm{mm}$ during exposure, which is below the value of $300 \mathrm{~mm}$ found for the PPR (Ebenholtz and Wolfson 1975). Sustained fixation to a target closer than this distance should elicit EMP inducing an increased tonic vergence (Ebenholtz 1974; Ebenholtz and Wolfson 1975; Paap and Ebenholtz 1977). Such a change in tonic vergence results in increased estimated distance. Binocular distance estimation is believed to rely on the departure from rest convergence rather than on absolute convergence (von Hofsten 1976). Any manipulation that changes rest convergence alters the effort required to fuse for all distances (Foley 1991). A shift of the rest convergence toward a shorter distance (i.e. an increase in tonic vergence) results in a reduced convergence effort, which leads to distance overestimation (Owens and Leibowitz 1980; Ebenholtz 1981; Ebenholtz b

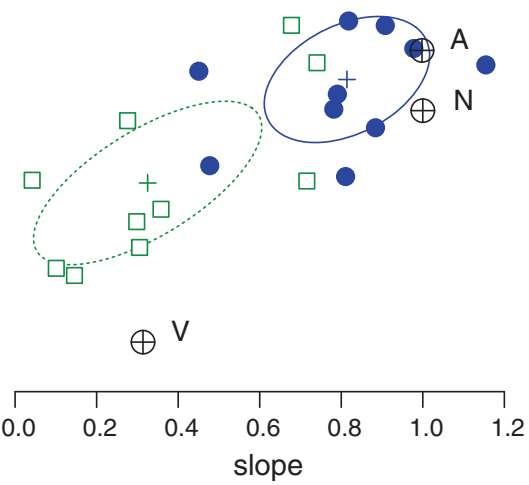

conditions in Exp. 1. The slope is represented in the horizontal axis and the mean pointing distance in the vertical axis. Each point corresponds to the values fitted for one subject in one of the two conditions, blue-filled circles for the pre-test phase and green open squares for the exposure phase. The 1-SD ellipses are shown (solid blue line for the pre-test and green dotted line for the exposure condition), as well as the mean value for each phase (crosses). Circles with crosses represent the theoretical values for the coefficients in each case $(V, A$ and $N$, as above)

and Fisher 1982; Shebilske et al. 1983; Fisher and Ciuffreda 1990). The second candidate for oculomotor adaptation is a change in accommodative vergence gain. The natural cross-coupling between accommodation and convergence is altered during telestereoscopic viewing and such a conflict has been found to be solved through an increased accommodative vergence gain (Miles et al. 1987; Bobier and McRae 1996). However, the use of a small light target in the present experiment reduced the accommodative stimulus, thus decreasing the accommodative drive to convergence as well as the accommodation and vergence mismatch. Some residual visual cues (such as accommodation, LED-size and LED-luminance) were present during the telestereoscopic exposure phase in Exp. 1. A calibration of the vergence/distance mapping may have been induced by a discrepancy between vergence-specified distance and residual cues signals for distance, leading to an increased slope of the target-to-pointing mapping. However, these weak residual visual cues are unlikely to have provided an efficient signal for distance.

According to Helmholtz's scaling theory, perception through a telestereoscope is such that "it will seem as if the observers were looking not at the natural landscape itself, but at a very exquisite and exact model of it", reduced in scale in the ratio IPD' to IPD (Helmholtz 1910). We did not obtain such a reduction of visual space during exposure phase in Exp. 1. The difference between observed and predicted estimated distances under telestereoscopic viewing may be explained by both the above considerations on oculomotor adjustments and a down-weighting of

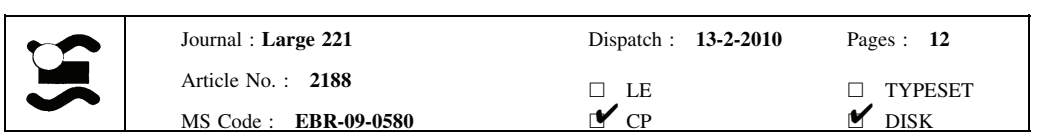


the contribution of vergence information. In the exposure condition, the estimated distances were greater than the vergence-specified distances, for all subjects. The training phase of binocular fusion took several minutes. It is likely that some EMP had already risen up during that period. Indeed, tonic vergence adaptation to prisms is known to start changing within the first minute of exposure (Schor 1979a; Hung 1992). Furthermore, a temporal analysis at the group level did not show any consistent effect of time on pointing response during the 10-min exposure condition. As described above, EMP might partially explain why the actual estimated distances were greater than those specified by vergence.

Pointing responses during exposure were actually located between vergence and accommodation-specified distances. This raises the possibility that different cues were combined with vergence for target distance estimate. Tresilian and Mon-Williams (1999) found that the presence of additional distance cues lowered the effects of the prism on perceived distance. In Judge and Bradford's experiment (1988), no confirmation of Helmholtz's scaling theory was found. These authors suggested that other cues may compete with binocular cues to modify the telestereoscope scaling factor. The influencing cues proposed in these two studies were monocular or binocular and were provided by the background scene or the changing size of the target. In the present study, even though most of the natural distance cues were eliminated, accommodation, LED-size and LED-luminance cues may have down-weighted vergence cues. The reliability of vergence information is indeed known to decrease with the amount of the discrepancy between vergence and other cues (Landy et al. 1995; Tresilian et al. 1999). Mon-Williams and Tresilian (2000) suggested that accommodation provides distance information through the accommodative vergence signal rather than through accommodation per se. Sustained exposure to an accommodative demand beyond the fixation distance may lead to increased tonic vergence (Schor 1979b) and thus greater estimated distances than as specified by vergence. However, the LED targets represented a poor stimulus to accommodation.

Interestingly, the mean slope (across subjects) of the target-to-pointing mapping under telestereoscopic viewing was not significantly different from the one predicted by using the vergence information according to Helmholtz's scaling theory. Further scrutiny of the individual data revealed a very large variability of the individual slopes.

\section{Effect of visuomotor exposure in Exp. 2}

The second and main goal of the present study was to examine the effect of visuomotor exposure on the plasticity of the vergence/distance mapping. A much larger
(2.3 times) aftereffect in distance estimation was found in Exp. 2 as compared to Exp. 1. In the same vein, Ebenholtz (1981) found a three-time greater aftereffect following a prism adaptation paradigm than following an induction exposure paradigm. Feedback was provided by both motor and visual monocular cues in Ebenholtz' adaptation paradigm.

The use of the hand contralateral to the exposed hand for distance estimation during pre- and post-test, without interhand contact in Exp. 2, prevented any potential hand proprioceptive or motor transfer. Thus, the hand pointing aftereffect of Exp. 2 can be considered as a reliable estimate of the visual distance aftereffect, uncontaminated by the short-term visuomotor adaptation of the exposed hand.

This visual aftereffect, in Exp. 2, involves two potential components: oculomotor adaptation and calibration of vergence/distance mapping. The former is assumed to be the same as in Exp 1. Indeed, target sequence, visual and oculomotor tasks were exactly the same in both experiments. The two experiments differed only by the visual feedback from the fingertip available during hand pointing in Exp. 2 but not in Exp. 1. Ebenholtz (1981) proposed that the amount of fusional stimuli present in the scene influences the aftereffect, EMP aftereffect increasing with greater stimulated retinal areas, as more disparity detectors are triggered. The vision of the LED on the left fingertip in Exp. 2 cannot be considered as an additional fusable stimulus as subjects were instructed to keep fixation onto the target during pointing. Moreover, it is unlikely that this additional LED point light provided a significantly different stimulus for accommodation. As Owens and Leibowitz (1980) and Owens (1986) found that interaction with a natural environment enhances the aftereffect due to EMP, care was taken in the current study to perform the pointing task in an otherwise dark room, the only visual stimuli being the fixated LED targets and the fingertip LED.

Since the contribution of oculomotor adaptation to the aftereffect is likely the same in both experiments, the increase in the aftereffect size from Exp. 1 to Exp. 2 may be explained by a calibration process. Calibration originates in the discrepancy between altered and veridical cues, the latter being either visual or coming from interaction with the environment (Wallach and Frey 1972; Wallach et al. 1972; Wallach and Smith 1972). Similarly, MonWilliams and Bingham (2007) have documented that reach distance is altered in response to distorted feedback (visual or haptic). Here, calibration of the vergence/distance mapping may arise from the discrepancy between altered vergence-specified distance and actual reach distance. As in Exp. 1, the residual visual cues present during the Exp. 2 exposure are unlikely to have provided an efficient signal for calibration of the vergence/distance mapping. During the Exp. 2 exposure, the target was seen closer than its

\begin{tabular}{|l|lll|}
\hline & Journal : Large 221 & Dispatch : 13-2-2010 & Pages : $\mathbf{1 2}$ \\
Article No. : $\mathbf{2 1 8 8}$ & $\square$ LE & $\square$ TYPESET \\
MS Code : EBR-09-0580 & $\sim_{\mathrm{CP}}$ & $\checkmark$ DISK \\
\hline
\end{tabular}


physical position. In the first trials in Exp. 2, subjects strongly undershot the target and had to make a secondary correcting movement to bring the fingertip LED in spatial coincidence with the target. As the target was a virtual image seen through the central half-silvered mirror, there was neither finger-to-target masking nor any tactile feedback. Finger size cues were also precluded as subjects only saw a point light on their fingertip.

As telestereoscopic viewing increases disparity, it modifies the perceived egocentric and target-to-fingertip relative distances, as well as the perception of motion in depth of the fingertip LED. There are different sources of error during the exposure condition: (1) an inconsistency between vision and proprioception of the fingertip, irrespective of the presence of a target (Craske and Crawshaw 1974), (2) an inconsistency between the expected fingertip LED visual feedback (derived from the efferent copy) and its actual visual feedback, irrespective of the presence of a target (Held and Hein 1958), (3) a terminal in-depth reaching error (Kitazawa et al. 1995; Magescas and Prablanc 2006) given by the increased disparity of the fingertip LED and (4) a discrepancy between the kinesthetic sensed hand motion and the resulting change in disparity of the fingertip LED. Increased disparity of the fingertip LED may have played a role during the end part of the movement only when the fingertip LED came into the narrow field of view through the telestereoscope (around $20^{\circ}$ ). Moreover, calibration of the vergence/distance mapping likely resulted from spatial inconsistency (items 1, 2 and 4 above) rather than from performance error (item 3) (Redding and Wallace 1997).

One potential issue is the extent to which the subjects were actually fusing the targets. Indeed, if the targets fell within Panum's area, subjects might have perceived the targets as single but without their eyes in alignment. If they were unable to accurately verge upon the targets this might have influenced the pattern of results. The tolerance range is Panum's fusion area, which is 15-30 arcmin (Ogle 1932; Schor et al. 1984). We calculated for the target range during exposure the maximum error in egocentric distance, which corresponds to a vergence error of 15-30 arcmin. This distance error increased with target distance, which means that there was a larger tolerance to fusion error for the greater distances. However, the maximum error was only $1.2-2.5 \mathrm{~mm}$ for the nearest target and $2.6-5 \mathrm{~mm}$ for the farthest target. Such errors can be considered as negligible.

A bias rather than a gain change for the calibration of the vergence/distance mapping in Exp. 2

The present telestereoscope paradigm involved exposure to an increased IPD. Calibration of the vergence/distance mapping induced by conflicting vergence-specified distance and actual reach distance was expected to lead to an increased slope (i.e. an increased gain) of the target-topointing mapping in Exp. 2. As a matter of fact, the posttest slope was not significantly different from that of the pre-test and a nearly constant bias was observed.

A possible interpretation is that change in gain of the vergence/distance mapping is not an inherent consequence of exposure to increased IPD. The nature of the aftereffect may depend on the exposure conditions. The lack of a distance-dependent effect may have been due to limitations on the exposure environment. First, we avoided rich uncontrolled environments in order to isolate the specific role of vergence in adaptation within reaching space. The poor visual environment limited the number of sources of error. Second, the assessment of distance perception was limited to reaching space in order to get an accurate measure of absolute distance with the most accurate method (i.e. by hand pointing). In addition, reaching space represents the locus of maximum interaction between perception and the oculomotor system. However, this restricted exposure range limited the strength of the distance-dependent error signals. Finally, the exposure duration was limited.

We found that a bias represented the adaptive response to such an optical distortion of vision under our experimental conditions. The obtained bias may be an economic way for the CNS to solve the conflict in the short term. Although a bias in the post-test might reflect a reduction of the conflict, the amount of adaptation was limited. Such a limited perceptual adaptation is comparable to that observed with short duration exposures to lateral prisms (Welch 1986).

A similar phenomenon was also observed by many authors (Fisher and Ciuffreda 1990; Bobier and McRae 1996) in the adaptation of the cross-couplings between vergence and accommodation. Exposure to an increased IPD calls for a change in cross-couplings gain (Miles et al. 1987). However, Fisher and Ciuffreda (1990) and Bobier and McRae (1996) obtained a bias in tonic vergence rather than a change in the accommodative vergence gain when the range of fixation distances was restricted. It indicates that both the nature and amount of the observed oculomotor adjustments depend on exposure conditions. Restriction of fixation distances during exposure seems to favor tonic adaptation (Miles et al. 1987; Bobier and McRae 1996). At the opposite, exposure to a constantly changing stimulus has been reported to reduce or prevent tonus adaptation (Paap and Ebenholtz 1977).

\section{Conclusion}

In the present study, exposure to telestereoscopic viewing was shown to produce a distance estimation aftereffect consisting of two components: a response to a sustained convergence demand onto the oculomotor system, and a

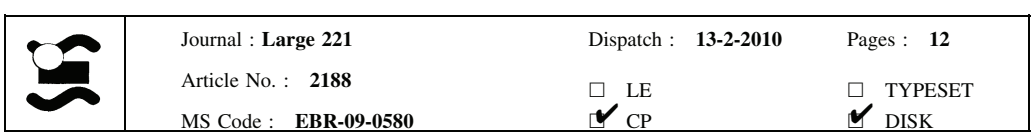


response to an inter-sensory conflict or to a conflict between expected and actual visual feedback. These two components were disentangled using different exposure paradigms. The calibration of the vergence/distance mapping resulting from distorted visual feedback consisted in a constant bias rather than the expected change in gain. Further studies are needed to determine whether the observed failure in a complete calibration is caused by limited distance exploration, too short exposure duration, or the nature and the intensity of the oculomotor/visuomotor conflict.

Acknowledgments The authors thank Véronique Chastres for help with the statistical analysis. They also thank Mark Mon-Williams and an anonymous reviewer for their helpful suggestions. The experiments were performed in the "Plateforme Mouvement et Handicap" HCL-IFNL (Lyon, France). This research was partly supported by grant No 07CO802 from Délégation Générale pour l'Armement.

\section{Appendix}

The distance estimated by pointing was assumed to be linearly correlated with both target distance and time. The coefficients were computed for the five open-loop conditions (pre- and post-tests in Exp. 1 and 2, and exposure in Exp. 1), according to the following model:

$y=a(t)+b(t) \times\left(x-x_{0}\right)$

where $t$ is the time elapsed since the beginning of the pretest, post-test or exposure conditions, $x$ is the target distance, $y$ is the pointing distance and $x_{0}$ is the mean value of the target distances $(430 \mathrm{~mm})$. Since the target distance mean value is subtracted from $x$, the term $a(t)$ corresponds to the mean pointing distance at a given instant $t$. The coefficient $b(t)$ is the instantaneous slope of the target-topointing mapping. A simple linear model is assumed for describing the temporal evolution of $a$ and $b$ :

$a(t)=a_{0}+a_{1}\left(t-t_{0}\right)$

$b(t)=b_{0}+b_{1}\left(t-t_{0}\right)$

where $t_{0}$ is the reference instant at the middle of each condition. The coefficients $a_{0}$ and $b_{0}$ correspond to the global mean values for the pointing distance and slope during the same condition. The time-variation coefficients $a_{1}$ and $b_{1}$ are related to the temporal evolution of the linear coefficients of target-to-pointing mapping.

\section{References}

Bingham GP, Pagano CC (1998) The necessity of a perception-action approach to definite distance perception: monocular distance perception to guide reaching. J Exp Psychol Hum Percept Perform 24:145-168
Blohm G, Khan AZ, Ren L, Schreiber KM, Crawford JD (2008) Depth estimation from retinal disparity requires eye and head orientation signals. J Vis 8:1-23

Bobier WR, McRae M (1996) Gain changes in the accommodative convergence cross-link. Ophthalmic Physiol Opt 16:318-325

Brown LE, Rosenbaum DA, Sainburg RL (2003) Limb position drift: implications for control of posture and movement. J Neurophysiol 90:3105-3118

Craske B, Crawshaw M (1974) Adaptive changes of opposite sign in the oculomotor systems of the two eyes. Q J Exp Psychol 26:106-113

Cutting JE (1997) High-Performance computing and Human vision I. Behav Res Methods Instrum Comput 29:27-36

Cutting JE, Vishton PM (1995) Perceiving layout and knowing distances: the integration, relative potency and contextual use of different information about depth. In: Epstein W, Rogers S (eds) Handbook of perception and cognition, vol 5. Perception of space and motion. Academic Press, San Diego, CA, pp 69117

Desmurget M, Epstein CM, Turner RS, Prablanc C, Alexander GE, Grafton ST (1999) Role of the posterior parietal cortex in updating reaching movements to a visual target. Nat Neurosci 2:563-567

Ebenholtz SM (1974) The possible role of eye-muscle potentiation in several forms of prism adaptation. Perception 3:477-485

Ebenholtz SM (1981) Hysteresis effects in the vergence control system: perceptual implications. In: Fisher DF, Monty RA, Senders JW (eds) Eye movements: visual perception and cognition. Erlbaum, Hillsdale, NJ, pp 83-94

Ebenholtz SM, Fisher SK (1982) Distance adaptation depends upon plasticity in the oculomotor control system. Percept Psychophys 31:551-560

Ebenholtz SM, Wolfson DM (1975) Perceptual aftereffects of sustained convergence. Percept Psychophys 17:485-491

Elliott D, Roy EA (1981) Interlimb transfer after adaptation to visual displacement: patterns predicted from the functional closeness of limb neural control centres. Perception 10:383-389

Fisher SK, Ciuffreda KJ (1990) Adaptation to optically-increased interocular separation under naturalistic viewing conditions. Perception 19:171-180

Foley JM (1977) Effect of distance information and range on two indices of visually perceived distance. Perception 6:449-460

Foley JM (1991) Binocular space perception. In: Regan D (ed) Vision and visual dysfunction vol 9. Binocular vision. Macmillan, London, pp 75-92

Genovesio A, Ferraina S (2004) Integration of retinal disparity and fixation-distance related signals toward an egocentric coding of distance in the posterior parietal cortex of primates. J Neurophysiol 91:2670-2684

Gogel WC, Tietz JD (1979) A comparison of oculomotor and motion parallax cues of egocentric distance. Vision Res 19:1161-1170

Goodale MA, Milner AD (1992) Separate visual pathways for perception and action. Trends Neurosci 15:20-25

Goodale MA, Pelisson D, Prablanc C (1986) Large adjustments in visually guided reaching do not depend on vision of the hand or perception of target displacement. Nature 320:748-750

Hamilton CR (1964) Intermanual transfer of adaptation to prisms. Am J Psychol 77:457-462

Hamilton CR, Bossom J (1964) Decay of prism aftereffects. J Exp Psychol 67:148-150

Harris CS (1963) Adaptation to displaced vision: visual, motor, or proprioceptive change? Science 140:812-813

Harris CS (1965) Perceptual adaptation to inverted, reversed, and displaced vision. Psychol Rev 72:419-444

Held R (1965) Plasticity in sensory-motor systems. Sci Am 213: 84-94

\begin{tabular}{|l|lll|}
\hline Journal : Large 221 & Dispatch : 13-2-2010 & Pages : $\mathbf{1 2}$ \\
Article No. : $\mathbf{2 1 8 8}$ & $\square$ LE & $\square$ TYPESET \\
MS Code : EBR-09-0580 & $\sim_{\text {CP }}$ & $\checkmark$ DISK \\
\hline
\end{tabular}


Held R, Freedman SJ (1963) Plasticity in human sensorimotor control. Science 142:455-462

Held R, Hein A (1958) Adaptation to disarranged hand-eye coordination contingent upon re-afferent stimulation. Percept Mot Skills 8:87-90

Howard IP, Rogers BJ (2002) Seeing in depth, vol 2. Depth perception I. Porteous, Toronto

Hung GK (1992) Adaptation model of accommodation and vergence. Ophthalmic Physiol Opt 12:319-326

Judge SJ, Bradford CM (1988) Adaptation to telestereoscopic viewing measured by one-handed ball-catching performance. Perception 17:783-802

Kitazawa S, Kimura T, Uka T (1997) Prism adaptation of reaching movements: specificity for the velocity of reaching. J Neurosci 17:1481-1492

Kornheiser AS (1976) Adaptation to laterally displaced vision: a review. Psychol Bull 83:783-816

Landy LS, Maloney LT, Johnston EB, Young M (1995) Measurement and modeling of depth cue combination: in defense of weak fusion. Vision Res 35:389-412

Martin TA, Keating JG, Goodkin HP, Bastian AJ, Thach WT (1996) Throwing while looking through prisms. II. Specificity and storage of multiple gaze-throw calibrations. Brain 119:11991211

Miles FA, Judge SJ, Optican LM (1987) Optically induced changes in the couplings between vergence and accommodation. J Neurosci 7:2576-2589

Mon-Williams M, Bingham GP (2007) Calibrating reach distance to visual targets. J Exp Psychol Hum Percept Perform 33:645-656

Mon-Williams M, Tresilian JR (1999) Some recent studies on the extraretinal contribution to distance perception. Perception 28:167-181

Mon-Williams M, Tresilian JR (2000) Ordinal depth information from accommodation? Ergonomics 43:391-404

Morton SM, Bastian AJ (2004) Prism adaptation during walking generalizes to reaching and requires the cerebellum. J Neurophysiol 92:2497-2509

Ogle KN (1932) An analytical treatment of the longitudinal horopter, its measurement and application to related phenomena, especially to the relative size and shape of the ocular images. J Opt Soc Am 22:665-728

Owens DA (1986) Oculomotor tonus and visual adaptation. Acta Psychol 63:213-231

Owens DA, Leibowitz HW (1980) Accommodation, convergence, and distance perception in low illumination. Am J Optom Physiol Opt 57:540-550

Paap KR, Ebenholtz SM (1977) Concomitant direction and distance aftereffects of sustained convergence: a muscle potentiation explanation for eye-specific adaptation. Percept Psychophys 21:307-314

Prablanc C, Martin O (1992) Automatic control during hand reaching at undetected two-dimensional target displacements. J Neurophysiol 67:455-469
Prablanc C, Tzavaras A, Jeannerod M (1975) Adaptation of the two arms to opposite prism displacements. Q J Exp Psychol 27:0105

Prablanc C, Desmurget M, Grea H (2003) Neural control of on-line guidance of hand reaching movements. Prog Brain Res 142:155170

Redding GM, Wallace B (1990) Effects on prism adaptation of duration and timing of visual feedback during pointing. J Mot Behav 22:209-224

Redding GM, Wallace B (1997) Adaptive spatial alignment. Lawrence Erlbaum Associates, Hillsdale, NJ

Schor CM (1979a) The influence of rapid prism adaptation upon fixation disparity. Vision Res 19:757-765

Schor CM (1979b) The relationship between fusional vergence eye movements and fixation disparity. Vision Res 19:1359-1367

Schor CM, Wood IC, Ogawa J (1984) Spatial tuning of static and dynamic local stereopsis. Vision Res 24:573-578

Tresilian JR, Mon-Williams M (1999) A curious illusion suggests complex cue interactions in distance perception. J Exp Psychol Hum Percept Perform 25:677-687

Tresilian JR, Mon-Williams M, Kelly BM (1999) Increasing confidence in vergence as a cue to distance. Proc Biol Sci 266:39-44

Valyus NA (1966) Stereoscopy. Focal Press, London

von Helmholtz H (1910) Handbuch der Physiologischen Optik. 3rd edition. Voss, Leipzig. English translation by JPC Southall (1925) Treatise on Physiological Optics: vol. 3. The perceptions of vision. Rochester NY: Optical Society of America. Electronic edition (2001): University of Pennsylvania

von Hofsten C (1976) The role of convergence in visual space perception. Vision Res 16:193-198

von Hofsten C (1979) Recalibration of the convergence system. Perception 8:37-42

Wade NJ (1998) A natural history of vision. MIT Press, Cambridge, MA

Wallace B, Redding GM (1979) Additivity in prism adaptation as manifested in intermanual and interocular transfer. Percept Psychophys 25:133-136

Wallach H, Frey K (1972) Adaptation in distance perception based on oculomotor cues. Percept Psychophys 11:77-83

Wallach H, Smith A (1972) Visual and proprioceptive adaptation to altered oculomotor adjustments. Percept Psychophys 11:413416

Wallach H, Frey K, Bode K (1972) The nature of adaptation in distance perception based on oculomotor cues. Percept Psychophys 11:110-116

Wann JP, Ibrahim SF (1992) Does limb proprioception drift? Exp Brain Res 91:162-166

Welch R (1986) Adaptation of space perception. In: Boff KR, Kaufman L, Thomas JP (eds) Handbook of perception and human performance, vol 1. Sensory process and perception. Wiley, New York

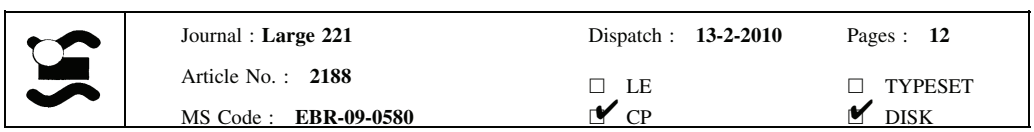

